\title{
Asymptotic formula for sum-free sets in abelian groups
}

\author{
by
}

R. Balasubramanian (Chennai) and Gyan Prakash (Allahabad)

Let $G$ be a finite abelian group of order $n$. A subset $A$ of $G$ is said to be sum-free if there is no solution of the equation $x+y=z$ with $x, y, z \in A$. Let $\operatorname{SF}(G)$ denote the set of all sum-free subsets of $G$, and $\sigma(G)$ denote the number $n^{-1} \log _{2}|\mathrm{SF}(G)|$. In this article we improve the error term in the asymptotic formula for $\sigma(G)$ which was recently obtained by Ben Green and Imre Ruzsa [GR05].

\section{DEFINITION 1.}

(I) Let $\mu(G)$ denote the density of a largest sum-free subset of $G$, so that any such subset has size $\mu(G) n$.

(II) Given a set $B \subset G$ we say that $(x, y, z) \in B^{3}$ is a Schur triple in $B$ if $x+y=z$.

Observing that all subsets of a sum-free set are sum-free we have the obvious inequality

$$
|\mathrm{SF}(G)| \geq 2^{\mu(G) n} .
$$

From (1) it follows trivially that $\sigma(G) \geq \mu(G)$.

In this article we improve the results of Ben Green and Imre Ruzsa [GR05] and prove Theorems 2 and 3 below. Theorem 2 follows immediately from Theorem 3 and [GR05, Proposition 2.1']. The methods used to prove Theorem 3 are a slight refinement of the methods in [GR05].

TheOREM 2. When $G$ is a finite abelian group of order $n$, then

$$
\sigma(G)=\mu(G)+O\left(\frac{1}{(\ln n)^{1 / 27}}\right) .
$$

THEOREM 3. There exists an absolute positive constant $\delta_{0}$ such that if $F \subset G$ has at most $\delta n^{2}$ Schur triples, where $\delta \leq \delta_{0}$, then

$$
|F| \leq\left(\mu(G)+c \delta^{1 / 3}\right) n,
$$

where $c$ is an absolute positive constant.

2000 Mathematics Subject Classification: 11B75, 20D60, $20 \mathrm{~K} 01$. 
Earlier Ben Green and Ruzsa [GR05] proved the following:

TheOREM 4 ([GR05, Theorem 1.8]). Let $G$ be a finite abelian group of order $n$. Then

$$
\sigma(G)=\mu(G)+O\left(\frac{1}{(\ln n)^{1 / 45}}\right) .
$$

Theorem 5 ([GR05, Proposition 2.2]). Let $G$ be a finite abelian group, and suppose that $F \subseteq G$ has at most $\delta n^{2}$ Schur triples. Then

$$
|F| \leq\left(\mu(G)+2^{20} \delta^{1 / 5}\right) n .
$$

The following theorem is also proven in [GR05].

TheOREM 6 ([GR05, Corollary 4.3]). Let $G$ be an abelian group, and suppose that $F \subseteq G$ has at most $\delta n^{2}$ Schur triples. Then

$$
|F| \leq\left(\max (\mu(G), 1 / 3)+3 \delta^{1 / 3}\right) n .
$$

Theorem 3 follows immediately from Theorem 6 in the case $\mu(G) \geq 1 / 3$. If $\mu(G)<1 / 3$, Theorem 3 again follows from Theorem 6 provided $\delta$ is not very small. For $\delta$ small we require Lemma 12 with an estimate different from those in [GR05]. For the rest of results required to prove Theorem 3, the methods used are completely identical as in [GR05], but the results used are not identical.

To prove Theorem 2 we use the following result from [GR05].

Theorem 7 ([GR05, Proposition 2.1']). Let $G$ be an abelian group of cardinality $n$, where $n$ is sufficiently large. Then there is a family $\mathcal{F}$ of subsets of $G$ with the following properties:

(I) $\log _{2}|\mathcal{F}| \leq n(\ln n)^{-1 / 18}$.

(II) Every $A \in \mathrm{SF}(G)$ is contained in some $F \in \mathcal{F}$.

(III) If $F \in \mathcal{F}$ then $F$ has at most $n^{2}(\ln n)^{-1 / 9}$ Schur triples.

Theorem 2 follows immediately from Theorems 7 and 3. We shall reproduce the proof given in [GR05]. If $n$ is sufficiently large as required by Theorem 7 then associated to each $A \in \mathrm{SF}(G)$ there is an $F \in \mathcal{F}$ for which $A \subset F$. For a given $F$, the number of $A$ 's which can arise in this way is at most $2^{|F|}$. Thus we have the bound

$$
|\mathrm{SF}(G)| \leq \sum_{F \in \mathcal{F}} 2^{|F|} \leq|\mathcal{F}| \max _{F \in \mathcal{F}} 2^{|F|} .
$$

Hence

$$
\sigma(G) \leq \mu(G)+C \frac{1}{(\ln n)^{1 / 27}}+\frac{1}{(\ln n)^{1 / 18}} .
$$

But from (1) we have $\sigma(G) \geq \mu(G)$. Hence Theorem 2 follows. 
In order to prove Theorem 3 we shall need the value of $\mu(G)$, which is now known for all finite abelian groups. In order to explain the results we make the following definition.

Definition 8. Suppose that $G$ is a finite abelian group of order $n$. If $n$ is divisible by any prime $p \equiv 2(\bmod 3)$ then we say that $G$ is of type $I$. We say that $G$ is of type $I(p)$ if it is of type I and if $p$ is the least prime factor of $n$ of the form $3 l+2$. If $n$ is not divisible by any prime $p \equiv 2(\bmod 3)$, but $3 \mid n$, then we say that $G$ is of type II. Otherwise $G$ is said to be of type III. That is, $G$ is of type III if and only if all divisors of $n$ are congruent to 1 modulo 3 .

The following theorem is due to P. H. Diananda and H. P. Yap [DY69] for type I and type II groups, and to Green and Ruzsa [GR05] for type III groups.

TheOREM 9 ([GR05, Theorem 1.5]). Let $G$ be a finite abelian group of order $n$. Then the following hold:

(I) If $G$ is of type $I(p)$ then $\mu(G)=1 / 3+1 / 3 p$.

(II) If $G$ is of type II then $\mu(G)=1 / 3$.

(III) If $G$ is of type III then $\mu(G)=1 / 3-1 / 3 m$, where $m$ is the exponent of $G$.

1. Cardinality of almost sum-free sets. In case the group $G$ is not of type III it follows from Theorem 9 that $\mu(G) \geq 1 / 3$ and hence Theorem 3 follows immediately using Theorem 6 . Therefore we have to prove Theorem 3 for type III groups only.

For the rest of this article $G$ will be a finite abelian group of type III, and $m$ will denote the exponent of $G$. The following proposition is an immediate corollary of Theorems 9 and 6.

Proposition 10. Let $G$ be an abelian group of type III with order $n$ and exponent $m$. If $F \subset G$ has at most $\delta n^{2}$ Schur triples then:

(I) $|F| \leq\left(\mu(G)+1 / 3 m+3 \delta^{1 / 3}\right) n$.

(II) If $\delta^{1 / 3} m \geq 1$ then $|F| \leq\left(\mu(G)+4 \delta^{1 / 3}\right) n$, that is, Theorem 3 holds in this case.

Therefore to prove Theorem 3 we are left with the following case: the group $G$ is an abelian group of type III with order $n$ and exponent $m$. The subset $F \subset G$ has at most $\delta n^{2}$ Schur triples and $\delta^{1 / 3} m<1$.

Let $\gamma$ be a character of $G$ and let $q$ be the order of $\gamma$. For any $j \in \mathbb{Z} / q \mathbb{Z}$, we define $H_{j}=\gamma^{-1}\left(e^{2 \pi i j / q}\right)$. We also denote the set $H_{0}=\operatorname{ker}(\gamma)$ by just $H$. Notice that $H$ is a subgroup of $G$, and $H_{j}$ are cosets of $H$ with cardinality 
$\left|H_{j}\right|=|H|=n / q$. For any set $F \subset G$ we also define $F_{j}=F \cap H_{j}$ and $\alpha_{j}=\left|F_{j}\right| /\left|H_{j}\right|$.

Proposition 11. Let $G$ be a finite abelian group of order $n$. Let $F$ be a subset of $G$ having at most $\delta n^{2}$ Schur triples where $\delta \geq 0$. Let $\gamma$ be any character of $G$ and $q$ be its order. Also let $F_{i}$ and $\alpha_{i}$ be as defined above. Then the following holds:

(I) If $x \in F_{i}$ and $y \in F_{j}$ then $x+y$ belongs to $H_{i+j}$.

(II) The number of Schur triples $\{x, y, z\}$ in $F$ with $x \in F_{l}, y \in F_{j}$ and $z \in F_{j+l}$ is at least $\left|F_{l}\right|\left(\left|F_{j}\right|+\left|F_{j+l}\right|-|H|\right)$. In other words, there are at least $\alpha_{l}\left(\alpha_{j}+\alpha_{j+l}-1\right)(n / q)^{2}$ Schur triples $\{x, y, z\}$ in $F$ with $x \in F_{l}$.

(III) Given any $l \in \mathbb{Z} / q \mathbb{Z}$ such that $\alpha_{l}>0$, we have

$$
\alpha_{j}+\alpha_{j+l} \leq 1+\delta q^{2} / \alpha_{l}
$$

for any $j \in \mathbb{Z} / q \mathbb{Z}$.

(IV) Given any $t \in \mathbb{R}$ we define

$$
L(t)=\left\{i \in \mathbb{Z} / q \mathbb{Z}: \alpha_{i}+\alpha_{2 i} \geq 1+t\right\} .
$$

Then

$$
\sum_{i \in L(t)} \alpha_{i} \leq \delta q^{2} / t
$$

Proof. (I) This follows immediately from the fact that $\gamma$ is a homomorphism.

(II) If $\left|F_{l}\right|\left(\left|F_{j}\right|+\left|F_{j+l}\right|-|H|\right) \leq 0$, there is nothing to prove. Hence we can assume that $F_{l} \neq \emptyset$. Then for any $x \in F_{l}$, we have $x+F_{j} \subset H_{j+l}$. Since also $F_{j+l} \subset H_{j+l}$ and $\left|F_{j}\right|+\left|F_{j+l}\right|-|H|>0$, it follows that

$$
\left|\left(x+F_{j}\right) \cap F_{j+l}\right|=\left|F_{j}\right|+\left|F_{j+l}\right|-\left|\left(x+F_{j}\right) \cup F_{j+l}\right| \geq\left|F_{j}\right|+\left|F_{j+l}\right|-|H| .
$$

Now for any $z \in\left(x+F_{j}\right) \cap F_{j+l}$ there exists $y \in F_{j}$ such that $x+y=z$. Hence the claim follows.

(III) From (II) there are at least $\alpha_{l}\left(\alpha_{j}+\alpha_{j+l}-1\right)(n / q)^{2}$ Schur triples in $F$. Hence the claim follows by the assumed upper bound on the number of those triples.

(IV) For any fixed $i \in L(t)$, taking $j=l=i$ in (II), we see that there are at least $\alpha_{i}(n / q)^{2} t$ Schur triples $\{x, y, z\}$ in $F$ with $x \in F_{i}$. Now for any $i_{1}, i_{2} \in L(t)$ such that $i_{1} \neq i_{2}$, the sets $F_{i_{1}}$ and $F_{i_{2}}$ are disjoint. Therefore there are at least $(n / q)^{2} t \sum_{i \in L(t)} \alpha_{i}$ Schur triples in $F$. Hence the claim follows.

Since the order of any character of an abelian group $G$ divides the order of the group and $G$ is of type III, the order $q$ of any character $\gamma$ of $G$ is odd and congruent to 1 modulo 3 . Therefore $q=6 k+1$ for some $k \in \mathbb{N}$. Let 
$I, H, M, T \subset \mathbb{Z} / q \mathbb{Z}$ denote the images of the intervals $\{k+1, k+2, \ldots, 5 k-1$, $5 k\},\{k+1, k+2, \ldots, 2 k-1,2 k\},\{2 k+1,2 k+2, \ldots, 4 k-1,4 k\},\{4 k+1$, $4 k+2, \ldots, 5 k-1,5 k\}$ in $\mathbb{Z} / q \mathbb{Z}$. Then the set $I$ is divided into $2 k$ disjoint pairs of the form $(i, 2 i)$ where $i \in H \cup T$.

Lemma 12. Let $G$ be a finite abelian group of type III and order $n$. Suppose that $F \subset G$ has at most $\delta n^{2}$ Schur triples. Let $\gamma$ be a character of $G$. Let the order of $\gamma$ be $q=6 k+1$. Then

$$
\sum_{i=k+1}^{5 k} \alpha_{i} \leq 2 k+2 \delta^{1 / 2} q^{3 / 2}
$$

Proof. The set $I=\{k+1, k+2, \ldots, 5 k\}$ is divided into $2 k$ disjoint pairs of the form $(i, 2 i)$ where $i \in H \cup T$. Therefore

$$
\sum_{i=k+1}^{5 k} \alpha_{i}=\sum_{i \in H \cup T}\left(\alpha_{i}+\alpha_{2 i}\right)
$$

Given a $t>0$ we divide $H \cup T$ into two disjoint sets,

$$
\begin{aligned}
& S=\left\{i \in H \cup T: \alpha_{i}+\alpha_{2 i} \leq 1+t\right\}, \\
& L=\left\{i \in H \cup T: \alpha_{i}+\alpha_{2 i}>1+t\right\} .
\end{aligned}
$$

Therefore

$$
\sum_{i \in H \cup T}\left(\alpha_{i}+\alpha_{2 i}\right)=\sum_{i \in S}\left(\alpha_{i}+\alpha_{2 i}\right)+\sum_{i \in L}\left(\alpha_{i}+\alpha_{2 i}\right) .
$$

From (7) we have

$$
\sum_{i \in L} \alpha_{i} \leq \delta q^{2} / t
$$

Since for any $l \in \mathbb{Z} / q \mathbb{Z}$, the inequality $\alpha_{l} \leq 1$ holds trivially, it follows that

$$
\sum_{i \in L}\left(\alpha_{i}+\alpha_{2 i}\right) \leq|L|+\delta q^{2} / t
$$

Also

$$
\sum_{i \in S}\left(\alpha_{i}+\alpha_{2 i}\right) \leq|S|+|S| t
$$

just by the definition of the set $S$. Now from (9), it follows that

$$
\sum_{i=k+1}^{5 k} \alpha_{i} \leq|L|+\delta q^{2} / t+|S|+|S| t \leq 2 k+q t+\delta q^{2} / t .
$$

Choosing $t=(\delta q)^{1 / 2}$ completes the proof of the lemma.

REMARK. The sum appearing in the last lemma was estimated by $2 k+$ $\delta^{1 / 2} q^{2}$ in [GR05]. There the estimate $\alpha_{i}+\alpha_{2 i} \leq \delta^{1 / 2} q$ was used to estimate the right hand side of (9). 
Notice that Lemma 12 holds for any character $\gamma$ of a group $G$ of type III. We would like to show that given $F \subset G$ having at most $\delta n^{2}$ Schur triples and also assuming that $\delta^{1 / 3} m<1$ where $m$ is the exponent of $G$, there is a character $\gamma$ such that $\alpha_{i} \leq c(\delta q)^{1 / 2}$ for $i \in\{0,1, \ldots, k\} \cup\{5 k+1, \ldots, 6 k\}$ where $c$ is an absolute positive constant, $q$ is the order of $\gamma$ and $k=(q-1) / 6$. To do this we recall the concept of special direction as defined in [GR05]. The method of proof of this part is identical as in [GR05], though the results are not.

Given any set $B \subset G$ and a character $\gamma$ of $G$ we define $\widehat{B}(\gamma)=\sum_{b \in B} \gamma(b)$. Fix a character $\gamma_{s}$ such that $\operatorname{Re} \widehat{B}(\gamma)$ is minimal. We follow the terminology in [GR05] and call $\gamma_{s}$ a special direction of $B$.

The following lemma is proven in [GR05], but we shall reproduce the proof here for the sake of completeness.

Lemma 13 ([GR05, Lemmas 7.1 and 7.3(iv)]). Let $G$ be an abelian group of type III. Suppose $F \subset G$ has at most $\delta n^{2}$ Schur triples. Let $\gamma_{s}$ be a special direction of $F$. Set $\alpha=|F| /|G|$. Then the following hold:

(I) $\operatorname{Re} \widehat{F}\left(\gamma_{s}\right) \leq\left(\frac{\delta}{\alpha(1-\alpha)}-\frac{\alpha^{2}}{\alpha(1-\alpha)}\right) n$.

(II) If $\delta \leq \eta / 5$, then either $|F| \leq \mu(G) n$ or

$$
q^{-1} \sum_{j=0}^{q-1} \alpha_{j} \cos \left(\frac{2 \pi j}{q}\right)+\frac{(\mu(\mathbb{Z} / q \mathbb{Z}))^{2}}{1-\mu(\mathbb{Z} / q \mathbb{Z})}<6 \delta .
$$

Proof. (I) There are exactly $n^{-1} \sum_{\gamma}(\widehat{F}(\gamma))^{2} \widehat{F}(\gamma)$ Schur triples in the set $F$. This follows after straightforward calculation, using the fact that

$$
\sum_{\gamma} \gamma(b)= \begin{cases}0 & \text { if } b \neq 0 \\ n & \text { if } b=0\end{cases}
$$

where 0 denotes the identity element of the group $G$. Therefore using the assumed upper bound on the number of Schur triples in $F$ it follows that

$$
n^{-1} \sum_{\gamma}(\widehat{F}(\gamma))^{2} \widehat{F}(\gamma)=n^{-1} \sum_{\gamma \neq 1}(\widehat{F}(\gamma))^{2} \widehat{F}(\gamma)+n^{-1}(\widehat{F}(1))^{2} \widehat{F}(1) \leq \delta n^{2},
$$

where $\gamma=1$ is the trivial character of $G$. Since $n^{-1}(\widehat{F}(1))^{2} \widehat{F}(1)=\alpha^{3} n^{2}$, it follows that

$$
\operatorname{Re} \widehat{F}\left(\gamma_{s}\right) \sum_{\gamma \neq 1}(\widehat{F}(\gamma))^{2} \leq n^{-1} \sum_{\gamma \neq 1}(\widehat{F}(\gamma))^{2} \widehat{F}(\gamma) \leq\left(\delta-\alpha^{3}\right) n^{2} .
$$

Since from (15) it follows that $\sum_{\gamma \neq 1}(\widehat{F}(\gamma))^{2}=\alpha\left(1-\alpha^{2}\right) n^{2}$, the claim follows. 
(II) We have $\operatorname{Re} \widehat{F}\left(\gamma_{s}\right)=|H| \sum_{j} \alpha_{j} \cos (2 \pi j / q)$. Therefore in the case $|F| \geq \mu(G)$, from (I) it follows that

$$
\begin{aligned}
& q^{-1} \sum_{j=0}^{q-1} \alpha_{j} \cos \left(\frac{2 \pi j}{q}\right) \leq \frac{\delta}{\alpha(1-\alpha)}-\frac{\alpha^{2}}{\alpha(1-\alpha)}, \\
& q^{-1} \sum_{j=0}^{q-1} \alpha_{j} \cos \left(\frac{2 \pi j}{q}\right)+\frac{(\mu(G))^{2}}{1-\mu(G)} \leq \frac{\delta}{\alpha(1-\alpha)} .
\end{aligned}
$$

Since from Theorem 9 we know that $\mu(G) \geq \mu(\mathbb{Z} / q \mathbb{Z})$ it follows that

$$
\frac{(\mu(G))^{2}}{1-\mu(G)} \geq \frac{(\mu(\mathbb{Z} / q \mathbb{Z}))^{2}}{1-\mu(\mathbb{Z} / q \mathbb{Z})} .
$$

The claim follows from this and the fact that $1 / 2 \geq \mu(G) \geq 1 / 4$, which implies that $\delta / \alpha(1-\alpha) \leq 6 \delta$.

Lemma 14. Let $G$ be an abelian group of type III with order $n$ and exponent $m$. Suppose $F \subset G$ has at most $\delta n^{2}$ Schur triples and $\delta^{1 / 3} m \leq 1$. Let $|F| \geq \mu(G) n$. Let $\gamma_{s}$ be a special direction of $F$ and $q$ the order of $\gamma_{s}$. Let $q=6 k+1$ and $\alpha_{i}$ be as defined above. There exist absolute positive constants $q_{0}$ and $\delta_{1}$ such that if $q \geq q_{0}$ and $\delta \leq \delta_{1}$, then

$$
\alpha_{i} \leq c(\delta q)^{1 / 2} \quad \text { for all } i \in\{0,1, \ldots, k\} \cup\{5 k+1, \ldots, 6 k-1\},
$$

where $c$ is an absolute positive constant.

Proof. If $F \subset G$ is as in the statement, then so is $-F \subset G$. Moreover $\left|F_{j}\right|=\left|(-F)_{-j}\right|$. Therefore to prove the proposition it is sufficient to show that

$$
\alpha_{i} \leq c(\delta q)^{1 / 2} \quad \text { for all } i \in\{0,1, \ldots, k\}
$$

for some absolute positive constant $c$.

Let

$$
S=q^{-1} \sum_{j=0}^{q-1} \alpha_{j} \cos \left(\frac{2 \pi j}{q}\right)+\frac{(\mu(\mathbb{Z} / q \mathbb{Z}))^{2}}{1-\mu(\mathbb{Z} / q \mathbb{Z})} .
$$

Then from Lemma 13 we have

$$
S \leq 6 \delta
$$

Now suppose that $\alpha_{l}>c(\delta q)^{1 / 2}$ for some $l \in\{0,1, \ldots, k\}$ (where $c$ is a positive number to be chosen later). We shall show that this violates (19), provided $q$ and $c$ are sufficiently large and $\delta$ is sufficiently small. For this we shall find the lower bound of $M=q^{-1} \sum_{j=0}^{q-1} \alpha_{j} \cos (2 \pi j / q)$.

Set $\gamma_{j}=\left(\alpha_{j}+\alpha_{j+l}\right) / 2$. Then we have

$$
M=\frac{1}{2 q \cos (\pi l / q)} \sum_{j=0}^{q-1} \alpha_{j}\left(\cos \left(\frac{(2 j+l) \pi}{q}\right)+\cos \left(\frac{(2 j-l) \pi}{q}\right)\right) .
$$


That is,

$$
M=\frac{1}{q \cos (\pi l / q)} \sum_{j=0}^{q-1} \gamma_{j} \cos \left(\frac{(2 j+l) \pi}{q}\right) .
$$

Notice that $\cos (\pi l / q)$ is not well defined if we consider $l$ as an element of $\mathbb{Z} / q \mathbb{Z}$. This is because the function $\cos (\pi t / q)$ as a function of $t$ is periodic, but with period $2 q$ and not $q$. But we have assumed that $l \in\{0,1, \ldots, k\}$, so the above computation is valid.

Since $\delta^{1 / 2} q^{3 / 2} \leq \delta^{1 / 2} m^{3 / 2}<1$ by assumption, recalling Lemma 11 it follows that

$$
2 \gamma_{j}=\alpha_{j}+\alpha_{j+l} \leq 1+\frac{1}{c} \delta^{1 / 2} q^{3 / 2} \leq 1+\frac{1}{c} \quad \text { for any } j \in \mathbb{Z} / q \mathbb{Z}
$$

and

$$
\sum_{j} \gamma_{j}=\sum_{j} \alpha_{j} \geq \mu(G) n \geq 2 k .
$$

The inequality (22) follows from the assumption that $|F| \geq \mu(G) n$.

Set $t_{c}=1+1 / c$. Let $E(c, q)$ denote the minimum of the expression $\sum_{j=0}^{q-1} \gamma_{j} \cos ((2 j+l) \pi / q)$ subject to the constraints $0 \leq \gamma_{j} \leq t_{c} / 2$ and $\sum_{j} \gamma_{j} \geq 2 k$.

The function $f: \mathbb{Z} \rightarrow \mathbb{R}$ given by $f(x)=\cos ((q+x) \pi / q)$ is even with period $2 q$ and

$$
f(0)<f(1)<\cdots<f(q) .
$$

Now to determine $E(c, q)$, we should choose $\gamma_{j}$ to be as large as we can when $\cos ((2 j+l) \pi / q)$ is small. We have two cases to discuss: when $l$ is even and when $l$ is odd. The image of the function $g: \mathbb{Z} / q \mathbb{Z} \rightarrow \mathbb{R}$ defined by $g(j)=\cos ((2 j+l) \pi / q)$ is equal to $\{f(x): x$ is even $\}$ if $l$ is odd, and to $\{f(x): x$ is odd $\}$ if $l$ is even. From this it is also easy to observe that the number of $j \in \mathbb{Z} / q \mathbb{Z}$ such that $\cos ((2 j+l) \pi / q)$ is negative is at most $(q+1) / 2$. Now let

$$
-\frac{q-1}{2}-l \leq j \leq \frac{q-1}{2}-l
$$

so that $-q \leq 2 j+l \leq q$. For $l$ odd consider the case when $\gamma_{j}=t_{c} / 2$ if

$$
2 j+l=q-\left[\frac{k}{t_{c}}-\frac{1}{2}\right], \ldots, q-2, q, q+1, \ldots, q+\left[\frac{k}{t_{c}}-\frac{1}{2}\right]
$$

and $\gamma_{j}=0$ otherwise. The condition $2\left[k / t_{c}-1 / 2\right]+1 \geq(q+1) / 2$ ensures that in the above configuration for all possible negative values of $\cos ((2 j+l) \pi / q)$ the maximum possible weight $t_{c} / 2$ is chosen. This condition can be ensured if $q \geq 11$ by choosing $c \geq c_{1}$ where $c_{1}$ is a sufficiently large absolute positive 
constant. Therefore a small calculation shows that for $c \geq c_{1}$,

$$
E(c, q) \geq-t_{c} \frac{\sin \left(2 \pi\left[k / t_{c}-1 / 2\right] / q\right)}{2 q \sin (\pi / q) \cos (\pi l / q)}-\frac{1}{q} .
$$

For $l$ even and $c \geq c_{1}$, choosing $\gamma_{j}=t_{c} / 2$ if

$$
2 j+l=q-\left[\frac{k}{t_{c}}\right], \ldots, q-1, q+1, \ldots, q+\left[\frac{k}{t_{c}}\right]
$$

and $\gamma_{j}=0$ otherwise, we get

$$
E(c) \geq-t_{c} \frac{\sin \left(\left(2 \pi\left[k / t_{c}\right]+1\right) / q\right)}{2 q \sin (\pi / q)} \cos \pi q-\frac{t_{c}}{q} .
$$

Using this we get

(26) $S \geq-t_{c} \frac{\sin \left(2 \pi\left[k / t_{c}\right] / q\right)}{2 q \sin (\pi / q) \cos (\pi l / q)}+\frac{(\mu(\mathbb{Z} / q \mathbb{Z}))^{2}}{1-\mu(\mathbb{Z} / q \mathbb{Z})} \quad$ when $l$ is even,

(27) $S \geq t_{c} \frac{\sin \left(2 \pi\left[k / t_{c}-1 / 2\right] / q\right)}{2 q \sin (\pi / q) \cos (\pi l / q)}-\frac{1}{q}+\frac{(\mu(\mathbb{Z} / q \mathbb{Z}))^{2}}{1-\mu(\mathbb{Z} / q \mathbb{Z})} \quad$ when $l$ is odd.

Now as $q \rightarrow \infty$ the right hand side of (26) as well as (27) converges to

$$
-t_{c} \frac{\sin \left(2 \pi / 3 t_{c}\right)}{2 \pi \cos (\pi l / q)}+\frac{1}{6}
$$

Let $\eta=2^{-20}$. Then choosing $c \geq c_{2}$ and $q \geq q_{0}$ and noticing that $l \leq q / 6$ we get

$$
S \geq-\frac{1}{2 \pi}+\frac{1}{6}-\eta=8 \delta_{1} \quad \text { say. }
$$

When $\delta \leq \delta_{1}$, the lower bound on $S$ given by (28) is in contradiction to the upper bound on $S$ given by (19). Hence the lemma follows.

To complete the proof of Theorem 3, we require the following result from [GR05].

Lemma 15 ([GR05, Proposition 7.2]). Let $G$ be an abelian group of type III and $n, m$ be its order and exponent respectively. Suppose $F \subset G$ has at most $\delta n^{2}$ Schur triples, with $\delta^{1 / 3} m<1$. Let $q$ be the order of a special direction such that $q \leq q_{0}$, where $q_{0}$ is an absolute positive constant as in Lemma 14. Also assume that $\delta \leq \eta / q^{5}=\delta_{2}$, where $\eta=2^{-50}$. Then either $|F| \leq \mu(G) n$ or $\alpha_{i} \leq 64 \delta^{1 / 3} q^{2 / 3}$ for any $i \in\{0,1, \ldots, k\} \cup\{5 k+1, \ldots, 6 k\}$.

Let $\delta_{1}$ and $\delta_{2}$ be as in Lemmas 14 and 15 respectively. Then we take $\delta_{0}=\min \left(\delta_{1}, \delta_{2}\right)$ in Theorem 3. Combining Lemmas 12, 14 and 15 yields Theorem 3 in case $\delta^{1 / 3} m<1$. In case $\delta^{1 / 3} m>1$, Theorem 3 follows from Proposition 10. 
Acknowledgments. The authors are very thankful to the anonymous referee for a careful reading of the first version of this paper and suggesting a number of improvements.

\section{References}

[DY69] P. H. Diananda and H. P. Yap, Maximal sum-free sets of elements of finite abelian groups, Proc. Japan Acad. 45 (1969), 1-5.

[GR05] B. J. Green and I. Z. Ruzsa, Sum-free sets in abelian groups, Israel J. Math. 147 (2005), 157-189.

The Institute of Mathematical Sciences CIT Campus, Taramani

Chennai 600113, India

E-mail: balu@imsc.res.in
Harish-Chandra Research Institute Chhatnag Road, Jhunsi

Allahabad 211019, India E-mail: gyan@mri.ernet.in

Received on 12.10.2005

and in revised form on 9.12.2006 